Chirurg 2011 $\cdot 82: 466$

DOI 10.1007/s00104-011-2112-x

Online publiziert: 20. April 2011

(c) Springer-Verlag 2011

\author{
J.R. Siewert ${ }^{1} \cdot$ V. Ewerbeck ${ }^{2}$ \\ ${ }^{1}$ Universitätsklinikum Heidelberg \\ ${ }^{2}$ Klinik für Orthopädie und Unfallchirurgie, Universitätsklinikum Heidelberg
}

\title{
Nachruf Professor Dr. Dr. h.c. Horst Cotta
}

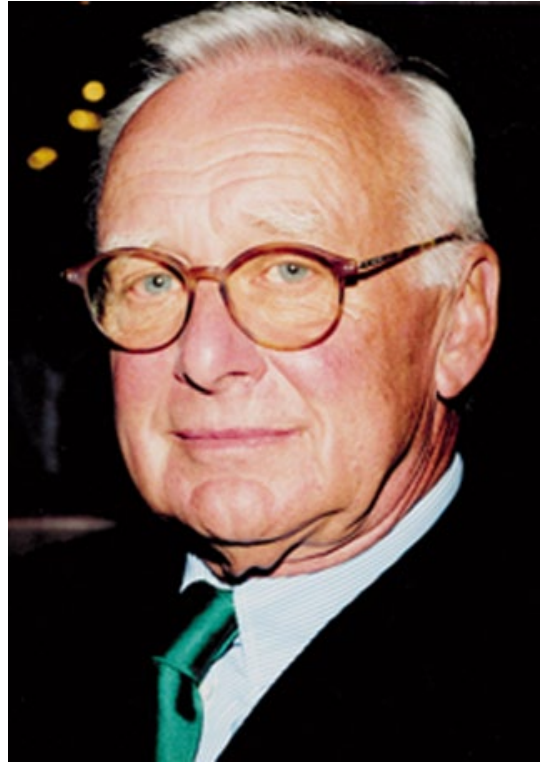

$\Delta$ Professor Dr. Dr. h.c. Horst Cotta, 1928-2011

Der Name Horst Cotta wird immer untrennbar mit der Orthopädischen Universitätsklinik Heidelberg verbunden bleiben. Von 1967 bis 1996 hat der gebürtige Berliner die ehemals „Orthopädische Anstalt" in die internationale Spitzengruppe der orthopädischen Universitätskliniken Europas geführt - sowohl durch eine Erweiterung des Behandlungsspektrums als auch durch maßgebliche wissenschaftliche Arbeiten, vor allem im Bereich des Gelenkverschleißes.

Horst Cotta wurde 1928 in Berlin geboren und blieb auch während seiner medizinisch-orthopädischen Ausbildung abgesehen von einem Studienaufenthalt in Zürich - seiner Heimatstadt treu. Auf der Grundlage seiner Ausbildung in der Pathologie und Chirurgie wandte er sich der Orthopädie zu und begann 1956 seine
Ausbildung im berühmten „Oskar-Helene-Heim “ der Orthopädischen Klinik der Freien Universität Berlin. (1961 erfolgte seine Habilitation.)

Im Jahr 1967 folgte er dem Ruf an die Universität Heidelberg, wo er mit dem Lehrstuhl für Orthopädie auch die „Stiftung Orthopädische Klinik“ als Direktor übernahm. Meilensteine seiner Tätigkeit in Heidelberg waren die Einführung der neuen Operationsmethoden beim chirurgischen Gelenkersatz der Intensivmedizin sowie der Aufbau der Versorgung querschnittgelähmter Patienten. Er gründete zudem die erste Abteilung für experimentelle Orthopädie in Deutschland, die sich mit Ursachen und Behandlungsmöglichkeiten der Arthrose auseinandersetzte. Unter seiner Führung wurden die Spezialambulanzen der Klinik deutlich erweitert, sodass Diagnostik und Therapie aller orthopädischen Erkrankungen von Spezialisten angeboten werden konnten. Renommierte Preise, Präsidentschaftenund Ehrenmitgliedschaften waren sichtbarer Ausdruck seiner wissenschaftlichen und klinischen Verdienste.

Auch in seinem Ruhestand nahm er noch großen Anteil an der Entwicklung seiner Fachdisziplin und seiner Klinik, aus der er zahlreiche Oberärzte in die Leitungspositionen orthopädischer Kliniken in ganz Deutschland entsenden konnte. Die Integration der Orthopädischen Universitätsklinik in das Universitätsklinikum Heidelberg 2009 hat Horst Cotta unterstützt und konstruktiv begleitet.

Einer breiteren Öffentlichkeit wurde Horst Cotta durch sein populäres Engagement für die Prävention orthopädischer Erkrankungen bekannt. Sein Rat- geber „Der Mensch ist so jung wie seine Gelenke“, der auf eigener Lebensführung aufbaute, machte ihn auch zum gefragten Ansprechpartner der Medien. Neben dem Sport waren die Künste sein Lebenselixier - Musik und seine Begeisterung für moderne Skulpturen, die Ausdruck in dem beeindruckenden Skulpturenpark der Klinik für Orthopädie fand und für sein Vermächtnis steht.

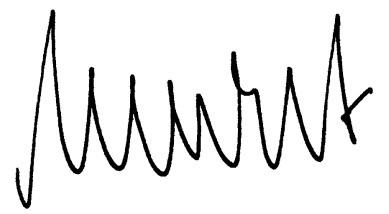

Prof. Dr. Dr. h.c. J.R. Siewert

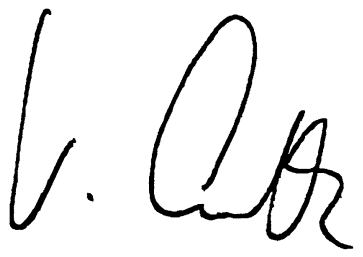

Prof. Dr. V. Ewerbeck

\section{Korrespondenzadresse}

Prof. Dr. Dr. h. c. J.R. Siewert

Universitätsklinikum Heidelberg, Im Neuenheimer Feld 672, 69120 Heidelberg JR.Siewert@med.uni-heidelberg.de 